\title{
Mini-Reoperative Mitral Valve Surgery
}

\author{
Angelo A. Vlessis, M.D., Ph.D., ${ }^{*}$ and Steven F. Bolling, M.D. ${ }^{\dagger}$ \\ ${ }^{*}$ Heart Institute, St. Vincent Hospital and Medical Center, Portland, Oregon, and ${ }^{t}$ \\ Section of Thoracic Surgery, University of Michigan, Ann Arbor, Michigan.
}

ABSTRACT Background: Reoperative surgery involving the atrioventricular valves places the patient at risk for cardiac or bypass graft injury upon reoperative sternotomy. Standard right thoracotomy can avoid these problems but is associated with a large incision and possibly more pulmonary complications. Methods and Results: An alternative, minimally invasive approach for reoperative atrioventricular valve surgery was studied in 22 patients. Patient age was $66 \pm 10$ years. Postoperative mitral regurgitation was $3.4 \pm 0.3$ and New York Heart Association (NYHA) Class was III/IV, despite a mean ejection fraction of $44 \pm 14 \%$. These patients had 1-4 prior procedures a mean of 5 years previously. An anterior 5th interspace incision of 5- to 10-cm was performed. A $1-\mathrm{cm}$ segment of 5 th rib was removed to facilitate exposure. Cardiopulmonary bypass was performed via ascending aorta or femoral artery cannula and bicaval venous cannulae. Systemic cooling $\left(25^{\circ}\right)$ and fibrillatory arrest was used. Operations performed included mitral valve repair (12), mitral valve replacement (5), prosthetic mitral valve rereplacement (4), repair of perivalvular leak (3), tricuspid valve repair (5), and atrial septal defect closures (7). Mean bypass time was $109 \pm 21$ minutes with a mean fibrillatory time of $62 \pm 12$ minutes. There was no intraoperative or 30-day mortality. Patients were weaned from ventilation at a mean of 5 hours postoperatively and received $1.3 \pm 1$ unit of blood. There were no wound complications or reexplorations for bleeding. At a mean follow-up of $15 \pm 8$ months, survivors are NYHA Class I-II. When interviewed, all patients felt their recovery was more rapid and less painful than their original sternotomy. Conclusion: This minimally invasive approach to reoperative atrioventricular valve surgery is safe and technically feasible. It has become our preferred approach to the atrioventricular valves in patients with a previous sternotomy. (J Card Surg 1998;13;468-470)

The potential dangers associated with reoperative sternotomy are well known to most cardiac surgeons. These reoperative candidates have aged and many have accumulated additional comorbid medical conditions since their previous cardiac operation. Moreover, valvular heart disease is accompanied by atrial dilation and cardiomegaly, which brings the thinned atrial wall in close apposition to the posterior sternum where it is poised for inadvertent injury during resternotomy. Additional risks include injury to patent bypass grafts, embolism of atherosclerotic debris dislodged from diseased aorta to patent bypass

Angelo A. Vlessis, M.D., Ph.D., St. Charles Hospital, 2500 N.E. Neff Road, Bend, OR 97701. Fax: 541-383-6825; e-mail: avlessis@yahoo.com grafts, as well as great vessel and phrenic nerve injury. These risks are often underestimated, but have been reported to be approximately $4 \%, 1,2$ Since 1990, we have successfully approached the atrioventricular valves in reoperative patients via a right thoracotomy. More recently, we have been able to significantly minimize the size of the incision by altering our surgical technique. This article describes our current technique and early experience with minimally invasive reoperative atrioventricular valve surgery at the University of Michigan Medical Center.

\section{METHODS}

Twenty-two patients underwent a minimally invasive right thoracotomy approach to the atrioventricular valves between December 1996 and 
October 1997 at the University of Michigan Medical Center. Patient demographic, operative, and postoperative data were collected prospectively.

\section{Operation}

Informed consent was obtained during the preoperative clinic visit and patients were admitted the morning of operation. After anesthetic induction, intubation was accomplished with a doublelumen endotracheal tube, and the patient was positioned for right anterolateral thoracotomy. External defibrillation pads were placed anterior and posterior on the chest wall out of the operative field. Skin preparation and draping proceeded in the usual sterile manner. A 5- to $10-\mathrm{cm}$ incision was made over the 5 th rib in the inframammary fold. The breast was elevated from the chest wall and the serratus anterior muscle elevated along its lateral border and partially divided inferiorly. The tatissimus dorsi muscle was retracted posteriorly. A $1-\mathrm{cm}$ segment of the 5 th rib was removed and the right lung collapsed to facilitate exposure. The pericardium was opened $1 \mathrm{~cm}$ anterior to the phrenic nerve from the superior vena cava to the inferior vena cava over the interatrial groove. The lung was covered with a moist lap, and stay sutures were placed along the posterior pericardial edge and brought out through the chest wall posterior to the incision under light traction. Arterial cannulation was accomplished in the ascending aorta (15 patients) or in the right femoral artery ( 7 patients). If necessary, a $1-\mathrm{cm}$ segment of 4 th rib was removed anteriorly, which greatly enhanced exposure of the aorta and simplified aortic cannulation. Bicaval venous cannulation was performed through the main incision using open-ended rightangled cannulae. On occasion, the inferior caval cannula was placed through a separate stab incision that was later used for a chest thoracostomy tube. After bypass was initiated, a left ventricular vent was placed through the right superior pulmonary vein and cooling commenced to $25^{\circ} \mathrm{C}$. The left atrium was opened after spontaneous fibrillation. The operation was performed on the perfused heart without clamping the aorta. Air was evacuated from the heart by allowing the left ventricle and left atrium to fill with blood dependently during left atrial closure. Residual air was removed with the aid of a left atrial and ascending aortic vent. Patients were rewarmed, weaned from bypass, and decannulated in the usual manner. In those patients who required atrial septal defect closure or tricuspid valve procedures in addition to a mitral valve procedure, the approach to the mitral valve was through the right atrium and the interatrial septum. Marcaine rib blocks were placed at chest closure to facilitate early postoperative extubation. A single 36-French chest tube was left for drainage.

\section{RESULTS}

\section{Demographics}

Patient age was $66 \pm 10$ years. Preoperative mitral regurgitation was $3.4 \pm 0.3$ on a scale of 1 to 4 by transesophageal echocardiography. NYHA Class was III/IV in all patients despite a preoperative ejection fraction of $44 \pm 14 \%$. Previous cardiac operations included coronary artery bypass grafting and aortic and mitral valve surgery. The number of previous cardiac operations ranged from 1 to 4 with a mean of 5 years since the last operation.

\section{Operation}

Operations performed included 12 mitral valve repairs, 5 mitral valve replacements, 4 mitral valve rereplacements, 3 repairs of periprosthetic leaks, 5 tricuspid valve repairs, and 7 atrial septal defect/patent foramen ovale closures. Mean cardiopulmonary bypass times were $109 \pm 21 \mathrm{~min}$ utes with a mean fibrillatory time of $62 \pm 12$ minutes. There was no intraoperative mortality.

\section{Postoperation}

Patients were weaned from ventilatory support at a mean of 5 hours postoperatively and required an average of $1.3 \pm 1.0$ units of blood. There were no wound complications or re-explorations for bleeding. No neurological or embolic complications were encountered. Thirty-day mortality was $0 \%$ with one late death from multiorgan failure. Although there was not a randomized, contemporaneous, "control" group, mean postoperative ICU, and total LOS were 1 day and 5.9 days, respectively. There was no incidence of postop low output syndrome or IABP use.

\section{Follow-up}

The duration of follow- up was $15 \pm 8$ months. Of the survivors, all were in NYHA Class I or II. 
When interviewed, all patients felt their recovery was more rapid and less painful than their previous sternotomy. There were no wound healing complications or infections.

\section{CONCLUSIONS}

Although right thoracotomy was one of the initial methods described for mitral valve surgery, most surgeons trained in the last 10 to 15 years have a limited experience with this approach and prefer median sternotomy for most atrioventricular valve operations. The patient with a previous sternotomy who requires atrioventricular valve surgery deserves special consideration because resternotomy is associated with additional risks not present in their initial operation. As such, several authors have demonstrated the utility of right thoracotomy for reoperative atrioventricular valve surgery in patients with a previous sternotomy. .-8 $^{-8}$ Most surgeons will agree that unintentional cardiac or bypass graft injury is reduced with the thoracotomy approach. In fact, decreased cardiopulmonary bypass times, blood loss, and blood product usage have been demonstrated in reoperative patients undergoing right thoracotomy as opposed to resternotomy. 3.4

Since 1990, we have been using the right thoracotomy approach to the isolated redo atrioventricular valves in patients with previous sternotomy. ${ }^{8}$ With the advent of minimally invasive techniques and a mind-set toward minimizing the surgical incision, alterations in our technique now enable us to safely perform reoperative atrioventricular valve surgery through a $5-$ to $10-\mathrm{cm}$ incision. All the excellent results and advantages of right thoracotomy over resternotomy previously presented ${ }^{8}$ are preserved, and the additional benefits of a smaller, more cosmetic incision and less postoperative discomfort are conferred.

Proper patient selection is paramount to success and safety. Patients who require aortic valve procedures and/or coronary artery bypass grafting in addition to atrioventricular valve surgery are better served with resternotomy. We have however, in two cases grafted the right coronary artery through a right thoracotomy, as well as performed redo mitral valve surgery, without difficulty. These patients did well. However, any aortic insufficiency can make exposure to the mitral valve difficult. A high profile Starr-Edwards aortic prosthesis usually has no aortic insufficiency with hypothermic fibrillation. However, the position of the cage apparatus must be kept in mind when cannulating the right lateral ascending aorta through the right chest approach. Although we have successfully used this mini-approach in patients with a previous right thoracotomy, significant scarring or right pleural disease may present a technical challenge. Proper patient selection and forethought can prevent intraoperative problems and surprises. If additional exposure is needed during the course of the operation, the incision is easily extended posterior or anterior across the sternum, although we have never found an anterior extension necessary.

In conclusion, our experience with an alternative, minimally invasive right anterolateral approach for reoperative atrioventricular valve surgery has been quite favorable. Patient satisfaction and comfort as well as surgeon satisfaction with safety and exposure are quite high. We continue to employ this approach at our institution and encourage other surgeons to consider its benefits.

\section{REFERENCES}

1. Husebye DG, Pluth JR, Piehler JM, et al: Reoperation on prosthetic heart valves: An analysis of risk factors in 552 patients. J Thorac Cardiovasc Surg 1983:86:543-552.

2. Lytle BW, Cosgrove DM, Taylor PC, et al: Reoperations for valve surgery: Perioperative mortality and determinants of risk for 1000 patients, 19581984. Ann Thorac Surg 1986;42:632-643.

3. Tribble CG, Killinger WA, Harman PK, et al: Anterolateral thoracotomy as an alternative to repeat sternotomy for replacement of the mitral valve. Ann Thorac Surg 1987;43:380-382.

4. Braxton JH, Higgins RDS, Schwann TA, et al: Reoperative mitral valve surgery via right thoracotomy: Decreased blood loss and improved hemodynamics. J Heart Valve Dis 1996;5:169-173.

5. Usui A, Kawamura M, Hibi NL, et al: Mitral valve replacement via right thoracotomy after coronary arterial grafting. Ann Thorac Surg 1995;60:708-709.

6. Cohn LH, Peigh PS, Sell J, et al: Right thoracotomy, femorofemoral bypass, and deep hypothermia for re-replacement of the mitral valve. Ann Thorac Surg 1989;48:69-71.

7. Berreklouw E, Alfieri O: Revival of right thoracotomy approach to the artrioventricular valves in reoperations. J Thorac Cardiovasc Surg 1984;32:331-333.

8. Steimle CN, Bolling SF. Outcome of reoperative valve surgery via right thoracotomy. Circulation 1996;94(Suppl II):II-126-II-128. 